\title{
Mobilizing political support proved critical to a successful switch from tOPV to bOPV in Kano, Nigeria 2016
}

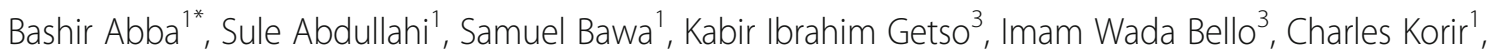
Audu Musa ${ }^{1}$, Fiona Braka ${ }^{1}$, Adamu Ningi ${ }^{1}$, Peter Nsubuga ${ }^{2}$, Richard Banda ${ }^{1}$, Sisay G. Tegegne ${ }^{1}$, Faisal Shuaib ${ }^{4}$, Usman Said Adamu ${ }^{4}$ and Sulaiman Haladu ${ }^{5}$

\begin{abstract}
Background: Kano is one of the high-risk states for polio transmission in Northern Nigeria. The state reported more cases of wild polioviruses (WPVs) than any other state in the country. The Nigeria Demographic and Health Survey of 2013 indicated that OPV3 coverage in the routine immunization (RI) programmewas 57.9\%. Additionally, serial polio seroprevalence studies conducted from 2011 to 2015 in the eightmetropolitan LGAs indicated low immunity levels against all three polio serotypes in children below one year. Areas with sub-optimal RI coverage such as Kanothat fail to remove all tOPV during the tOPV-bOPV switchwill be at increased risk of VDPV2 circulation.

Methods: We assessed the impact of political leadership engagement in mobilizing other stakeholders on the outcomes of the bOPV-tOPV switch in Kano State from February to May 2016 using nationally-selected planning and outcome indicators.

Results: A total of 670 health facilities that provide RI services were assessed during the pre-switch activities. Health workers were aware of the switch exercise in 520 (95.1\%) of the public health facilities assessed. It was found that health workers knew what to do should tOPV be found in any of the 521 (95.2\%)public health facilities assessed. However, there was a wide disparity between the public and private health practitioners' knowledge on basic concepts of the switch.

There was $100 \%$ withdrawal of tOPV from the state and the seven zonal cold stores. Unmarked tOPVwas found in the cold chain system in 2 (4.5\%) LGAs. Only one health facility $(0.8 \%)$ had tOPV in the cold chain. No tOPVwas identified outside the cold chain without the "Do not use" sticker in any of the health facilities.

Conclusion: The engagement of the political leadership to mobilize other key stakeholders facilitated successful implementation of the tOPV-bOPVswitch exercise and provided opportunity to strengthen partnerships with the private health sector in Kano State.
\end{abstract}

Keywords: Mobilization, Stakeholder, tOPV-bOPV switch, Circulating vaccine-derived poliovirus, Kano

\footnotetext{
* Correspondence: abbab@who.int

${ }^{1}$ World Health Organization, Country Representative Office, Abuja, Nigeria

Full list of author information is available at the end of the article
}

(c) The Author(s). 2018 Open Access This article is distributed under the terms of the Creative Commons Attribution 4.0 International License (http://creativecommons.org/licenses/by/4.0/), which permits unrestricted use, distribution, and reproduction in any medium, provided you give appropriate credit to the original author(s) and the source, provide a link to the Creative Commons license, and indicate if changes were made. The Creative Commons Public Domain Dedication waiver (http://creativecommons.org/publicdomain/zero/1.0/) applies to the data made available in this article, unless otherwise stated. 


\section{Background}

Poliomyelitis is an acute paralytic disease caused by three polioviruses (PV) serotypes. Less than $1 \%$ of PV infections result in acute flaccid paralysis [1]. The Sabin oral polio vaccine (OPV) and the inactivated polio vaccine (IPV) are used for active immunization against the disease. In 1988, when $>350,000$ persons in 125 countries were affected by polio, the 41st World Health Assembly resolved to eradicate the disease by the year 2000 [2].

While OPV was instrumental in the success recorded in Polio Eradication Initiative (PEI), OPV has the disadvantage of genetic instability, resulting in cases of vaccine-associated paralytic poliomyelitis (VAPP) and vaccine-derived polioviruses(VDPVs) [3]. The potential risk of circulating vaccine-derived poliovirus (cVDPV) emergence has increased in recent years as wild poliovirus circulation has ceased in most of the world. The risk appears highest for the type 2 OPV strain because of its greater tendency to spread to contacts [4].

In 2013, the World Health Assembly endorsed a plan that calls for the ultimate withdrawal of OPV from all immunization programs globally. The withdrawal was to be conducted in a phased manner with the removal of the type 2 component of OPV in 2016 through a global switch from trivalent OPV to bivalent OPV [5]. IPV is introduced into routine immunization (RI) schedules in all countries to reduce the risk for cVDPV2 outbreaks and to facilitate responses to outbreaks. By the end of September 2015, 90 (46\%) of 194 World Health Organization (WHO) member states were using IPV [6].

Nigeria made significant progress in polio eradication in recent years with the removal of the country from the list of endemic countries in September, 2015 by WHO. In April 2016, Nigeria participated in the switch exercise wheretOPVwas replaced with bOPV, the second objective of the Polio Eradication and Endgame Strategic Plan, 2013-2018 [7, 8].

Kano is one of the key high-risk states for polio transmission in Northern Nigeria. The state is also the most populous in the country. The state reported more cases of wild polioviruses (WPVs) than any other in the country. Kano has made significant progress in polio supplemental immunization activities (SIA) over the last few years. The proportion of Local Government Areas (LGAs) that attained coverage of $80 \%$ and above (assessed bylot quality assurance sampling (LQAS) surveys) rose from $60 \%$ in 2013 to $97 \%$ in 2016. However, this progress is precarious, because the Nigeria Demographic and Health Survey of 2013 indicated that OPV3 coverage in the routine immunization (RI) programme was 57.9\% [9]. Also, serial polio seroprevalence studies conducted from 2011 to 2015 in the eightmetropolitan LGAs indicated low immunity levels against all three polio serotypes in children below 1 year [10]. Strong RI is essential for immunity against PV2 after the switch since IPV, delivered by RI program, would be the only source of PV2 antigen. Areas with sub-optimal RI coverage such as Kano will, therefore, be at increased risk of VDPV circulation. Accordingly every efforts should be made to remove all tOPV from all facilities to minimize this risk.

The interplay between politics and immunization in Kano is known to play a role in suspension of Polio Vaccination [11]. Misunderstandings and inadequate communication led Kano state to boycott polio immunization in 2003-2004 with devastating consequences to the global PEI [12]. The issue of vaccine derived polio virus was carefully handled and communicated to the public in the state. Immunization sceptics propagated the rumour that OPV was unsafe [13]. Therefore, any misinformation on the withdrawal of tOPV to prevent VDPV could play into the hands of these sceptics. Kano state health authorities felt there was a need for transparency, but the information should be passed in such a way that it contains no unnecessary details to spark controversy [14]. Additionally, there was a wide range of stakeholders handling tOPV in the state. Major stakeholders were private medical practitioners, pharmaceutical companies,and security agencies. Political leadership was required to effectively engage them for assured and complete withdrawal of tOPV. Thus, this paper describes the process of political leadership engagement of various stakeholders, the role played by individual stakeholders and the impact of this collaboration in the successful implementation of the switch between February and April 2016 in Kano.

\section{Methods}

We conducted a cross-sectional descriptive study in Kano state, northern Nigeria from February to May 2016.

In doing so we targeted the leadership of Kano State Ministry of Health, the National Agency for Food and Drug Administration and Control (NAFDAC) and health professional associations that have representation in the state, public and private health facilities that provide RI service.

The National bOPV-tOPVSwitch Committee recommended the establishment of state switch committees to coordinate planning and implementation of the switch plan. The committee in Kano was headed by the Incident Manager (IM) at the State Emergency Operations Center (EOC). Members included state team leads of partner agencies:WHO, United Nations Children's Fund (UNICEF), US Centers for Disease Control (CDC), Rotary International, Core-Group, and European Union Support to immunization Governance in Nigeria (EU-SIGN). The state switch committee established the state switch support team which comprised representatives of state primary health care development board (SPHCMB) and all partner agencies. The support team developed a detailed switch plan for the state and supported its implementation. Similar support teams were established in all the 44 LGAs. 
The state switch committee paid an advocacy visit to the state Health Commissioner and briefed him on the switch plans and solicited for his active participation in the switch process. He was specifically requested to lead in the media engagement and other stakeholder engagement. The Health Commissioner convened series of meetings with representatives of private health practitioners where he highlighted the importance of the switch process and specific actions required from them. These included granting access to supervisors, allowing the withdrawal of any tOPV that may have been found and operating beyond their normal working hours if required. The Health Commissioner also issued a directive to all public health facilities in the state to ensure availability of RI focal persons even outside working hours in the week of the switch. Additionally, letters on the switch were written to commandants of all military and paramilitary health institutions in the state as well as weekly review meetings held on the challenges and the solution to the switch process under the Health Commissioner.

We informed key stakeholders about the switch process. The stakeholders included local government area (LGA) primary health care coordinators, medical directors of state general hospitals, private medical practitioners and the pharmaceutical society of Nigeria. NAFDAC which regulates pharmaceutical products was requested to be part of the supervision team to pharmaceutical companies and drug dealers that stock tOPV.

We trained all the personnel who participated in the switch data collection process. Data collection tools were developed at the national EOC. State and LGA level trainings were synchronized with SIA training for March 2016. On the job training for health facility staff were conducted during supervision by state supervisors. However, the training for independent switch validators was conducted by the national EOC. The switch had three stages: (1) pre-switch inventory of tOPV stock, (2) tOPV withdrawal and replacement with bOPV and (3) post switch validation. The pre-switch inventory stage involved identification and documentation of all tOPV stock, assessment of personnel's knowledge on the switch and cold chain facilities. State pre-switch supervisors were identified from government and partner agencies.

Questionnaires were applied at LGA stores, satellite coldstores, and health facilities. Immediate feedback on the outcome of the supervision was provided at all levels. Weekly review meetings at state and LGA levels were conducted to discuss challenges and identify solutions.

The withdrawal stage involved removal of tOPV stock within and outside the cold chain. The withdrawn tOPV were marked "Do Not Use." The Health Commissioner held a media briefing on the day of the switch to intimate the public on the switch and its objectives. The main purpose of the briefing was to pre-empt any mischievous misrepresentation on the dangers of tOPV that may be attempted by immunization skeptics. The validation stage involved inspection of all state, zonal and LGA cold stores for the presence of tOPV and bOPV. Similarly, health worker knowledge on the switch was assessed. The post switch validation was conducted from 19th to 21st April 2016 by independent monitors who were recruited outside the immunization system.

Data from the advocacy activities were summarized in MS word, while data on pre-switch supervision and post switch validation were collated and processed using MS Excel software package.

\section{Results}

The Kano state Ministry of Health provided a clear leadership in the switch process. The Health Commissioner led the media engagement which was considered critical taking into account the sensitivity and the misunderstanding that often surround PEI interventions by the media in the state. Part of media engagement activities conducted by the commissioner was a media briefing on the switch day which attracted public and private media representatives. The commissioner highlighted objectives of the switch and the participants were given anopportunity to ask questions and seek clarifications on any issue about the switch.

NAFDAC provided valuable support during the planning and implementation of the switch. The agency provided information on pharmaceutical companies and other drug dealers that handled tOPV. Their staff also participated in the supervision of the pharmaceutical companies during pre-switch preparation which facilitated access to the companies (Table 1 ). The agency also supervised the destruction of 27,351 retrieved tOPV vials by the boil-and-bury method as outlined in the National Switch Committee guidelines.

A total of 670 health facilities (547 public and 123 private) that provide RI services were assessed during the pre-switch activities. Pre-switch assessment was conducted by teams of at least two persons. A total of 123 staff from government and partners participated in the exercises. Information collected during the visits, in addition to physical verification of tOPV withdrawal, included health facility staff knowledge on the switch and what to do should tOPV is found in the cold chain system. The result of the pre-switch assessment showed that 496 (91\%) public health facilities had active cold chain while only 61 (49\%) of private health facilities had active cold chain. Health workers were aware of the switch exercise in 520 (95.1\%) of the public health facilities assessed. The health workers knew what to do should tOPV be found in any of the 521 (95.2\%) of public health facilities assessed. The involvement of the private medical practitioners facilitated a hitch-free supervision 
Table 1 Summary of advocacy activities conducted and results on tOPV-bOPV switch in Kano State, April 2016

\begin{tabular}{lll}
\hline Stakeholder targeted & Commitment secured & Implementation \\
\hline Health & Meet with private health practitioners to solicit & The HC met with representatives of private health practitioners on \\
Commissioner (HC) & for cooperation on switch & Tpril 2016 \\
& $\begin{array}{l}\text { Brief media on the switch plans } \\
\text { Direct public health workers to operate outside } \\
\text { working hours during the switch week }\end{array}$ & $\begin{array}{l}\text { All public health workers were directed to be at their workplace even } \\
\text { outside working hours during the switch }\end{array}$ \\
& $\begin{array}{l}\text { Letter to security bodies to allow access to their } \\
\text { facilities for the switch }\end{array}$ & $\begin{array}{l}\text { Letterswere written to medical directors and commandants of Army, } \\
\text { Airforce, Police and Immigration Service hospitals in Kano to grant access }\end{array}$ \\
& $\begin{array}{l}\text { Monitor implementation of switch plans } \\
\text { to their facilities and allow retrieval of any tOPV that may have been found }\end{array}$ & $\begin{array}{l}\text { HC Chaired weekly review meeting on switch with the state switch } \\
\text { committee }\end{array}$ \\
& $\begin{array}{l}\text { Participate in pre-switch supervision of } \\
\text { pharmaceutical and major drug dealers that }\end{array}$ & $\begin{array}{l}\text { Provided two representatives who participated as state switch monitors } \\
\text { that visited pharmaceutical and drug dealers that stock tOPV }\end{array}$ \\
& stock tOPV & \\
\hline
\end{tabular}

and validation of private health facilities. However, there was a wide disparity between the public and private health practitioners' knowledge on basic concepts of switch (Fig. 1).

A total of 54 cold stores were assessed during the post-switch validation process: one state, seven zonal and 44 LGA. All the 54 (100\%) cold stores assessed had both active and passive cold chain. There was $100 \%$ withdrawal of tOPV from the state and the seven zonal cold stores. However, tOPVwas found in the cold chain system in 2 (4.5\%) LGAs (Table 2).

A total of 122 health facilities were validated by the independent monitors. The monitors found tOPV in the cold chain system of one $(0.8 \%)$ health facility. However, there was no tOPV identified outside the cold chain without the "Do not use" sticker. Only $77.8 \%$ of the health facilities had bOPV and 79.5\% had IPV(Table. 3). The findings indicate that the state had met the minimum performance required to be certified as to have successfully switched from tOPV to bOPV.

\section{Discussion}

Our study found that political leadership involvement in planning and implementation of the tOPV-bOPV switch in Kano State was critical to the success of the exercise. The engagement of these stakeholders by the leadership of the ministry of health facilitated their active participation in the switch. The roles played by NAFDAC and Pharmaceutical Council of Nigeria were particularly important in obtaining vital information on private

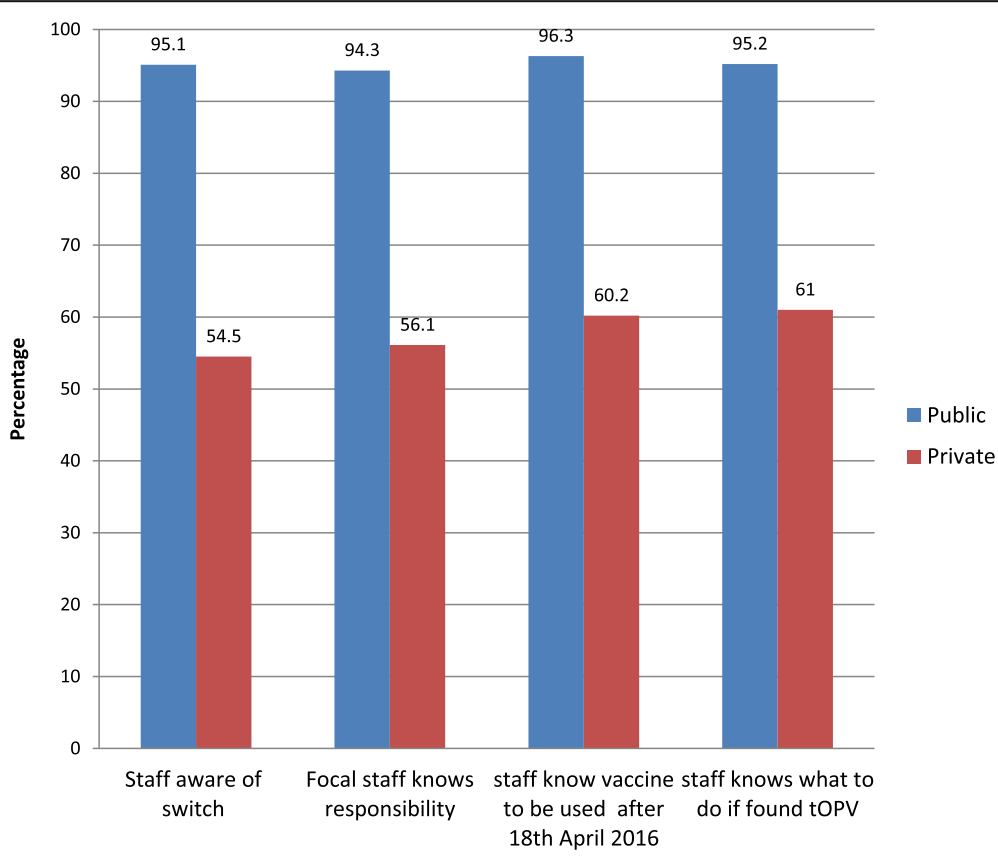

Fig. 1 Knowledge of public and private health facility staff on switch in Kano, April 2016 
Table 2 Switch Validation of cold stores and health facilities by Independent Monitors in Kano, April 2016

\begin{tabular}{|c|c|c|c|c|}
\hline \multirow[t]{3}{*}{ Indicators } & \multicolumn{2}{|c|}{ State \& Zonal Stores } & \multicolumn{2}{|c|}{ LGA Stores } \\
\hline & \multicolumn{2}{|l|}{ Yes } & \multicolumn{2}{|l|}{ Yes } \\
\hline & No & $\%$ & No & $\%$ \\
\hline Has active cold chain & 8 & 100 & 44 & 100 \\
\hline Has passive cold chain & 8 & 100 & 44 & 100 \\
\hline tOPV in cold chain & 0 & 0 & 2 & 4.5 \\
\hline tOPV out of cold chain without label "Do not use" sticker & 0 & 0 & 0 & 0 \\
\hline bOPV available & 8 & 100 & 44 & 100 \\
\hline IPV available & 8 & 100 & 44 & 100 \\
\hline
\end{tabular}

pharmaceutical outfits that stock vaccines and in getting access to inspect them. Our findings re-affirmed that enlisting political support and building partnerships are key areas that need to be addressed for successful implementation of public health interventions [15]. Post switch monitoring revealed zero tOPV at the state and seven zonal satellite cold stores; this issignificant as finding tOPV in these facilities after the switch would have posedhigh riskin the distribution of tOPV to health facilities. In all, two (4.5\%) LGA cold stores were found to have had tOPV that was not appropriately labeled as "Do Not Use". More importantly, of the 122 health facilities monitored after the switch, only one $(0.8 \%)$ had tOPV.

Although the tOPVwas withdrawn from the cold chain systems at the facility level, $77.8 \%$ had stock of bOPV. Also, $79.5 \%$ of the health facilities assessed had IPV. The absence of IPV in more than $20 \%$ of the health facilities after the switch is of particular concern. IPV was introduced into the RI schedule before the switch to reduce the risk of reintroduction of type 2poliovirus [6].

We also found a wide disparity in the understanding of the switch between public and private health facility staff. Only $54.5 \%$ of private health workers interviewed were aware of the switch as against $95.1 \%$ in public facilities. Also, $60.2 \%$ of workers in private facilities knew which polio vaccine to be used after the switch as against $96.3 \%$ in public facilities. This apparent low knowledge of private health workers on current polio

Table 3 Switch validation of cold chain at health facilities by independent monitors in Kano, April 2016

\begin{tabular}{lll}
\hline Indicators & Health Facilities \\
\cline { 2 - 3 } & Yes & $\%$ \\
\hline tOPV in Cold Chain & 1 & 0.8 \\
tOPV out of cold chain & 0 & 0 \\
without label "Do not & & \\
use" sticker & & 77.9 \\
bOPV available & 95 & 79.5 \\
IPV available & 97 & \\
\hline
\end{tabular}

immunization guidelines corroborates the widely held concern on the quality of care provided by private facilities in low-income countries [16].

The limitations of this study include the timing of visits to health facilities during the pre-switch supervision. As often is the case in public and private health facilities, a particular health worker is identified as the RI focal person, hence the risk of interviewing health worker with inadequate training in RI. Also, the post-switch validation involved formal RI facilities. However, there could still be private health facilities offering RI that source their vaccines elsewhere which were not necessarily known to the system.

Despite those potential limitations, the engagement of political leadership to mobilize other key stakeholders had facilitated successful implementation of the switch exercise in Kano. We will, therefore, recommend sustained advocacy to the political leadership for increased guidance in enlisting the support of critical stakeholders, private and public, in planning, implementation, monitoring and supervision of public health interventions.

\section{Conclusion}

Nigeria switched from tOPV to bOPV in April 2016. This study demonstrated that the engagement of the political leadership to mobilize other key stakeholders facilitated successful implementation of the tOPV-bOPV switch exercise and provided opportunity to strengthen partnerships with the private health sector in Kano State.

\section{Abbreviations}

bOPV: Bivalent oral polio vaccine; CDC: US Centers for Disease Control; cVDPV: Circulating vaccine-derived poliovirus; EOC: Polio emergency operations center; EU-SIGN: European Union Support to immunization Governance in Nigeria; IM: Incident Manager; IPV: Inactivated polio vaccine; LGA: Local government area; LQAS: Lot quality assurance sampling; MS Excel: MicrosoftExcel; NAFDAC: National Agency for Food and Drug Administration and Control; OPV: Oral polio vaccine; OPV3: Oral polio vaccine 3rd dose; PEl: Polio Eradication Initiative; PV: Polio viruses; Rl: Routine immunization; SIA: Supplemental immunization activities; SPHCMB: State Primary HealthCare Development Board; tOPV: Trivalent oral polio vaccine; UNICEF: United Nations Children's Fund; VAPP: Vaccine-associated paralytic poliomyelitis; VDPV2: Vaccine Derived Polio Virus Type 2; WHO: World Health Organization; WPV: Wild polio virus 


\section{Acknowledgements}

We acknowledge the support of WHO country Office for their encouragement and continued guidance. We also appreciate the support of Kano Polio Emergency Operation Centre and State Primary Health Care Management Agency.

\section{Funding}

Publication of this article was sponsored by the grant from WHO, Nigeria country office.

\section{Availability of data and materials}

The data sets analyzed during this study is available from Kano State Polio Emergency Operations Centre, Ministry of Health Kano, Kano State. The data is available from Imam Wada Bello.

\section{About this supplement}

This article has been published as part of BMC Public Health Volume 18 Supplement 4, 2018: Experiences and lessons learned in polio eradication in Nigeria. The full contents of the supplement are available online at https:// bmcpublichealth.biomedcentral.com/articles/supplements/volume-18supplement-4.

\section{Authors' contributions}

BA was the lead author, participated in data analysis and wrote the drafts. PN and SA supported in formulating the study design and reviewed the initial drafts. FB, CK, USA, and FS participated in the development of health facility and cold store questionnaires. KIG supported advocacy data collection. SB reviewed and participated in the analysis of data on advocacy. IWB provided the data on switch validation. SH participated in training of monitors and field supervision of health facility data collection. AM and AN participated in the review of the manuscripts. RB and SGT reviewed the draft, participated in data analysis and tracked the implementation of editors' corrections of the drafts. All authors read and approved the final manuscript.

\section{Ethics approval and consent to participate}

\section{Not Applicable.}

The data analyzed for this study was collected as part of the Synchronized tOPV/bOPV switch implementation by the Kano state ministry of health. Selected facilities were visited by the validators to collect the relevant information pertaining to the switch, including interviews with health workers. The Kano State Ministry of Health Ethics Committee on Research considered that the study does not involve human or animal samples, invasive or any other manipulative procedures.

\section{Consent for publication}

Not applicable

Data does not include identifiable individual information (names, pictures etc). The health workers freely participated as part of program information collection to be analyzed and used.

\section{Competing interests}

The authors declare that they have no competing interests.

\section{Publisher's Note}

Springer Nature remains neutral with regard to jurisdictional claims in published maps and institutional affiliations.

\section{Author details}

${ }^{1}$ World Health Organization, Country Representative Office, Abuja, Nigeria. ${ }^{2}$ Global Public Health Solutions, Atlanta, GA, USA. ${ }^{3}$ Ministry of Health, Kano, Kano State, Nigeria. ${ }^{4}$ National Primary Health Care Development Agency, Abuja, Nigeria. ${ }^{5}$ Africa Field Epidemiology Network, Hospital Road, Kano, Kano State, Nigeria.

\section{Published: 13 December 2018}

\section{References}

1. Baicus A. History of polio vaccination. World J Virol. 2012;1(4):108.

2. World Health Organization. Forty-First World Health Assembly: Global eradication of poliomyelitis by the year 2000. 1988. p. 2000. Available from: http://www.who.int/ihr/polioresolution4128en.pdf. Accessed 25 July 2016.
3. Burns CC, Diop OM, Sutter RW, Kew OM. Vaccine-derived polioviruses. J Infect Dis. 2014:210:5283-93.

4. Kew OM, Wright PF, Agol VI, Delpeyroux F, Shimizu H, Nathanson N, Pallansch MA. Circulating vaccine-derived polioviruses: current state of knowledge. Bull World Health Organ. 2004;82(1):16-23.

5. Patel M, Zipursky S, Orenstein W, Garon J, Zaffran M. Polio endgame: the global introduction of inactivated polio vaccine. Expert Rev Vaccines. 2015; 14(5):749-62 Available from:https://www.researchgate.net/publication/ 271219713_Polio_endgame_The_global_introduction_of_inactivated_polio_ vaccine. Accessed 25 July 2016.

6. Immunization Systems Management Group of the Global Polio Eradication Initiative. Introduction of inactivated poliovirus vaccine and switch from trivalent to bivalent Oral poliovirus vaccine - worldwide, 2013-2016. MMWR. Morb Mortal Wkly Rep. 2015;64(25):699-702 Available from: http://www. ncbi.nlm.nih.gov/pubmed/26135591. Accessed 25 $5^{\text {th }}$ July 2016.

7. P. Minor, "The polio endgame.," Hum Vaccin Immunother., vol. 10, no. 7, pp. 2106-2108, 2014. Available from: http://www.pubmedcentral.nih.gov/ articlerender.fcgi?artid=4370356\&tool=pmcentr. Accessed on 27 ${ }^{\text {th }}$ July 2016.

8. Sutter RW, Platt L, Mach O, Jafari H, Aylward RB. The new polio eradication end game: rationale and supporting evidence. J Infect Dis. 2014;210:S434-8.

9. N. P. Commission. Nigeria demographic and health survey 1999. MD Natl Popul Comm ORC. 2013:59.

10. K. T. Craig, H. Verma, Z. lliyasu, P. Mkanda, K. Touray, T. Johnson, A. Walla, R. Banda, S. G. Tegegne, Y. G. Yehualashet, B. Abba, A. Ahmad-Shehu, M. Takane, R. W. Sutter, P. Nsubuga, A. J. G. Muhammad, and R. G. M. Vaz, "Role of Serial Polio Seroprevalence Studies in Guiding Implementation of the Polio Eradication Initiative in Kano, Nigeria: 2011-2014," J Infect Dis, p. jiv774, 2016. Available from: http://jid.oxfordjournals.org/lookup/doi/10.1093/ infdis/jiv774. Accessed on $25^{\text {th }}$ July 2016.

11. Kaufmann JR, Feldbaum H. Diplomacy and the polio immunization boycott in northern Nigeria. Health Aff. 2009;28(4):1091-101.

12. Obadare E. A crisis of trust: history, politics, religion and the polio controversy in northern Nigeria. Patterns Prejudice. 2005;39(3):265-84.

13. Ghinai I, Willott C, Dadari I, Larson HJ. Listening to the rumours: what the northern Nigeria polio vaccine boycott can tell us ten years on. Glob Public Health. 2013;8(10):1138-50 Available from: http://www.pubmedcentral.nih. gov/articlerender.fcgi?artid=4098042\&tool=pmcentrez\&rendertype=abstract. Accessed $22^{\text {nd }}$ August 2016.

14. Yahya M. Polio vaccines--'no thank you!' barriers to polio eradication in Northern Nigeria. Afr Aff (Lond). 2006;106(423):185-204 Available from: http://afraf.oxfordjournals.org/cgi/doi/10.1093/afraf/adm016. Accessed on $22^{\text {nd }}$ August 2016

15. Frieden TR. Six components necessary for effective public health program implementation. Am J Public Health. 2014;104(1):17-22.

16. Mills A, Brugha R, Hanson K, McPake B. What can be done about the private health sector in low-income countries? Bull World Health Organ. 2002;80(4): $325-30$.
Ready to submit your research? Choose BMC and benefit from:
- fast, convenient online submission
- thorough peer review by experienced researchers in your field
- rapid publication on acceptance
- support for research data, including large and complex data types
- gold Open Access which fosters wider collaboration and increased citations
- maximum visibility for your research: over $100 \mathrm{M}$ website views per year
At BMC, research is always in progress.
Learn more biomedcentral.com/submissions 